\title{
Pharmaceutical development of biologics: fundamentals, challenges and recent advances
}

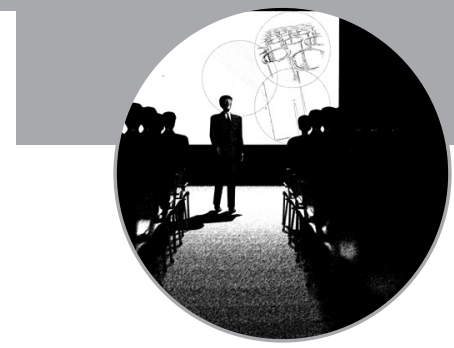

\author{
AAPS 46th Arden Conference \\ 6-10 March 20II, West Point, NY, USA
}

The 46th Arden Conference, held in West Point, NY, USA, March 20II, focused on development of protein therapeutics, comprising preformulation, formulation, manufacturing, advanced delivery systems, protein characterization/analysis, and regulatory landscape. The sessions of preformulation and formulation development consisted of nine lectures discussing protein stability implications and characterization during purification, freeze-drying and manufacturing. The session on advanced drug delivery encompassed two new sustained-release microsphere formulations (protein microencapsulation by annealing of premade porous PLGA microspheres and aqueous-aqueous emulsion for preformulating proteins to solvent-resistant particles), two transdermal insulins (patching after thermal ablation of skin and phase-transition hydrogel microneedle patch), and a responsible hydrogel system for intra-ear delivery. The sessions on analytical technologies and regulatory landscape both focused on challenges for biosimilars.

\section{Global pharmaceutical pipeline \& development challenges}

The conference was started with a Keynote address delivered by John D Ludwig (Pfizer Biotherapeutics Pharmaceutical Sciences, MA, USA) regarding global trends, technological challenges and future opportunities of the industry. According to Ludwig, biotherapeutics will comprise $50 \%$ of 100 top products by 2014, approximately $16 \%$ annual growth from 2008's 28\%. By 2016, eight of the predicted top ten drugs are likely to be biologics. Among biotherapeutics, $65 \%$ of sales are coming from recombinant proteins, $24 \%$ from monoclonal antibodies, 9\% from vaccines, and RNAi and tissue engineering products fill the rest of the market. The rapid growth of the biologic market is driven by the increasing importance of biotherapeutics, entry of the biosimilars, bioprocessing technology advances and new therapeutics modalities.

\section{Drug substances development \& preformulation development}

Larry Dumont (BioScience Consulting Services, CA, USA) reviewed almost all the aspects of biodrug substance production, comprising expression system and cell-line development, bioreactor optimization, purification optimization, final drug-substance composition and storage, and drug-substance characterization and analysis. Among them, analytical methods are pivotal throughout research, process development, formulation development, forced degradation study and manufacturing quality control. For expression systems, Dumont provided a comprehensive description on mammalian cell-line selection and development.

Stephen Byrn (Purdue University, IN, USA) lectured on the chemistry of protein drug substances in a solid state comprising of the stabilization effects of glassy and crystalline phases and water content of protein formulations. Analytic methods to characterize solidstate drug products were reviewed. In brief, the degree of hydration of a solid-state protein drug plays a crucial role in stabilizing and maintaining protein native form and bioactivity. A 'fully' hydrated (20-40\% water content) yet still solid and glassy form should be the ideal environment for maintaining protein stability.

\section{Formulation \& manufacturing process}

Arvind Srivastava (ImClone Systems, NY, USA) reviewed the implications of protein stability on formulation and product development, comprising of physical and chemical instability, formulation parameters implicating protein stability in dosage form preparation and manufacturing process. Michael Pikal (University of Connecticut, CT, USA) had a more focused lecture on the freeze-drying process of proteins. Pikal reviewed the processes of water removal in freeze-drying, and discussed the relevance of relaxation dynamics in glasses with protein stability. According to Pikal, pharmaceutical stability in solids is not driven by thermodynamics. The thermal denaturation of proteins in solids is as high as $130-190^{\circ} \mathrm{C}$, a temperature substantially higher than that for protein degradation. Due to this
Jing Su', Jeffrey Mazzeo², Nanda Subbarao ${ }^{3}$ \& Tuo Jin ${ }^{\dagger 1}$

'School of Pharmacy, Shanghai Jiao Tong University, Shanghai, China ${ }^{2}$ Waters Corporation, Boston, MA, USA

${ }^{3}$ Biologics Consulting Group, Inc., New York City, NY, USA ${ }^{\dagger}$ Author for correspondence: E-mail: tjin@sjtu.edu.cn 


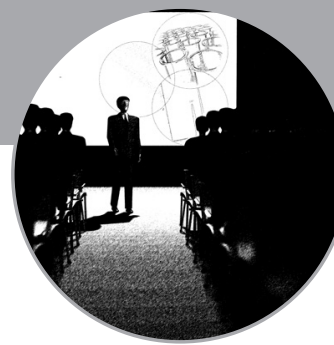

phenomenon, protein stabilizers, such as disaccharides, which prevent proteins from degradation but at the same time lower thermal denaturation temperature may be used. The lecture did not include polysaccharides as protein stabilizers in the freeze-drying process for their ability to offer a hydroxyl-rich environment and their resistance to glass phase transition.

Steven Shire (Genentech, Inc., CA, USA) presented two lectures on formulation strategy of high concentration monoclonal antibodies (mAbs) and the impact of aggregation on safety and physical properties of protein biotherapeutics. Due to the need of a large dose, concentration-associated issues, such as aggregation,

\section{Opportunities for aggregation:}

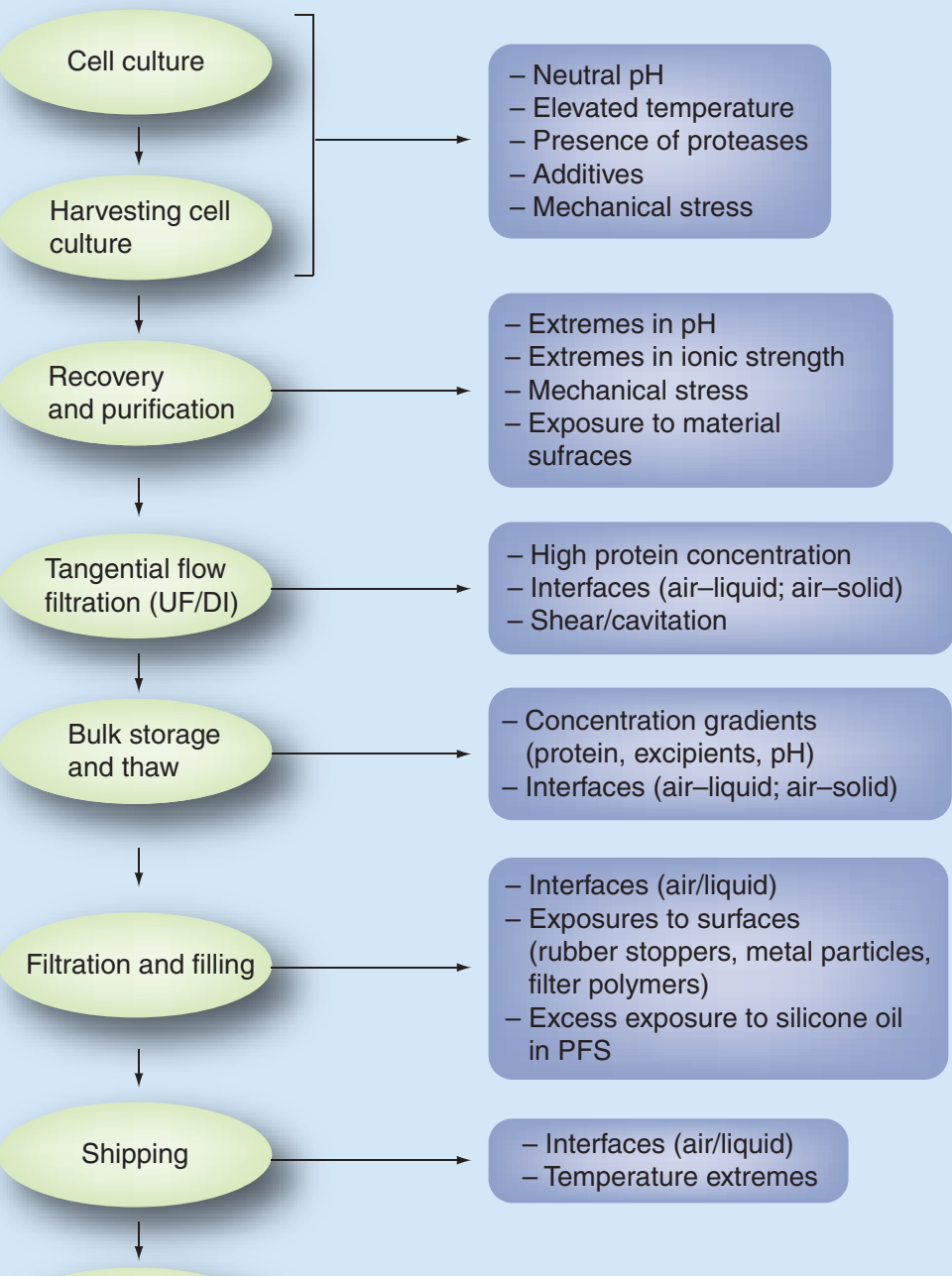

End users

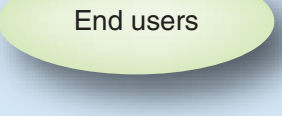

Figure 1. Manufacturing of biomolecules via unit operations.

Adapted with permission from [1]. viscosity, stability and analysis, become great challenges for $\mathrm{mAb}$ formulation, production and quality control. These issues need to be addressed from a variety of aspects comprising formulation design, $\mathrm{mAb}$ design, delivery device design and manufacturing process design. Protein aggregation in a pharmaceutical dosage form, either in soluble or precipitate forms, is a major safety concern in developing biotherapeutics. Since $90 \%$ of protein-native epitopes are non-continuous, aggregates and particulates may form new epitopes and evoke antibodies that cross-react with the body's native protein monomers. There are many opportunities for proteins to aggregate from expression, purification, formulation, manufacturing, shipping and storage to end users (Figure I) [1]. It is not yet clear what pathways of the immune responses to aggregates is the most important. Preventing or limiting aggregation to an acceptable, safe, level is the only solution today.

Carol Kirchhoff (Pfizer, NY, USA) updated quality by design for biopharmaceutical formulation and process development, comprising design space, risk assessments and control strategy. Biopharmaceutical packaging and container selection were discussed by Michael Akers (Baxter, IL, USA).

\section{Advanced delivery systems}

For sustained-release delivery of unaltered native proteins, Steven Schwendeman (University of Michigan, MI, USA) introduced a newly developed technology for organic solvent-free microencapsulation of protein drugs. Preparation of sustained-release microspheres involved organic solvents that are not only hazardous for protein stability but also complicate manufacturing processes by the requirement to include sterilization conditions and organic solvent evacuation in a unit operation. The technology developed by the Schwendeman laboratory involves an annealing process of pre-manufactured poly(lactic-coglycolic) acid (PLGA) microspheres possessing sufficient pores. The porous PLGA microspheres may be manufactured in large scale without a sterilization condition. The microspheres may then be impregnated in a protein solution so that the protein may be absorbed in the microspheres under organic-solvent-free sterilized conditions, followed by a thermal annealing process above the glassy transition temperature of PLGA to seal the pores. The draw back of this approach may be that the capacity of the absorption-based protein loading may be limited. To enlarge protein loadings, aluminum hydroxide powders were 
pre-loaded in the porous PLGA microspheres to adsorb protein therapeutics. However, proteins not being denatured by adsorption on the surfaces of aluminum oxide should be selected.

Tuo Jin (Shanghai Jiao Tong University, Shanghai, China) demonstrated their aqueousaqueous emulsion for pre-loading proteins into polysaccharide fine particles without contacting water-oil or water-air interfaces, factors known to denature proteins (FIGURE 2) [2]. Delicate proteins loaded in the glassy polysaccharide particles become tolerant to organic solvents and, thus, can safely be suspended in a PLGA solution and formulated to microspheres, fibers and medical device coatings without denaturing [3]. The encapsulated polysaccharide particles may also protect proteins during the prolonged sustained release period by forming a viscous phase around the macromolecules when absorbing body fluid at the injection site. The content of the polysaccharide loaded in the microspheres may be an easily tunable parameter to adjust protein release kinetics. As a shortcoming of this process, a clean room with solvent evacuation is needed for formulation.

Oral delivery of biologics may be the most challenging task in formulation development. Raphael Mannino (University of Medicine and Dentistry of New Jersey, NJ, USA) presented cochleates, a lipid bilayer spiral roll, for oral delivery of amphotericin B (AmB) [4]. Cochleates consist of negatively charged phospholipid (phosphatinyl serine) bilayers by interacting with divalent ions (such as calcium or zinc ions), which function as ionic bridging reagents between the two anionic arrays of lipid bilayers. As a particulate system structured of phospholipid bilayers, a cochleate differs from a liposome in that it has a solid interior and mechanical strength derived from this rigidity. Moreover, the lipid bilayer of a cochleate possesses edges at the two ends of the roll whose tension may serve as a driving force for the particle to fuse with the cell membrane and release its cargos into the cell. $\mathrm{AmB}$ is an ellipsoid-shaped molecule with one side hydrophilic and the other side hydrophobic and a length slightly shorter than the thickness of the lipid bilayer. Several of the molecules may form a cluster with the hydrophobic side facing the lipids and perfectly 'sits' in the bilayer matrix. Mannino also showed some preliminary yet interesting results of using cochleates to deliver siRNA. Cochleated siRNA labeled with Cy3 were imaged as fluorescent dots, $<1 \mu \mathrm{m}$ in size; and anti-influenza siRNA loaded in nanocochleates showed an in vivo efficacy in $\mathrm{BALB} / \mathrm{c}$ mice 200-fold higher than naked ones. To encapsulate negatively charged siRNA into cochleates formed of negatively charged phosphatidyl serine (PS), the researchers mixed some cationic lipids into PS prior to cochleation. A hypothesized structure of the siRNA-cochleate particle was that siRNA formed an complex with cationic lipids, which sits in the hydrophobic matrix of the PS bilayer of a cochleate. It will be more convincing if a method of nanometer-resolution could be used to characterize the proposed structure and to confirm that siRNA is encapsulated in cochleates. Similarly for the efficacy study, a comparison with pure cationic lipids (such as lipofectamine-loaded antisense siRNA), instead of naked antisense siRNA or cochleated nonsense siRNA would be interesting.

William Lambert (Pacira Pharmaceuticals, Inc., NJ, USA) discussed the evolution, types and applications of liposomes as drug-delivery systems. Liposomes were first described by Bangham in 1961, and up to 6614 research papers were published between 2000 and 2008. Types of liposomes include unilamella, multilamella and multivesicular forms, and their targeted delivery functions were improved from plain liposomes to stealth liposomes, targeting moiety-conjugated stealth liposomes and responsive drug-release liposomes. Applications of liposomal dosage forms are found in the delivery of antifungal drugs, nucleotide drugs and proteins [5].

For transdermal delivery of insulin, Eric Tomlinson (Altea Therapeutics, GA, USA) described the usage of a thermal ablator developed

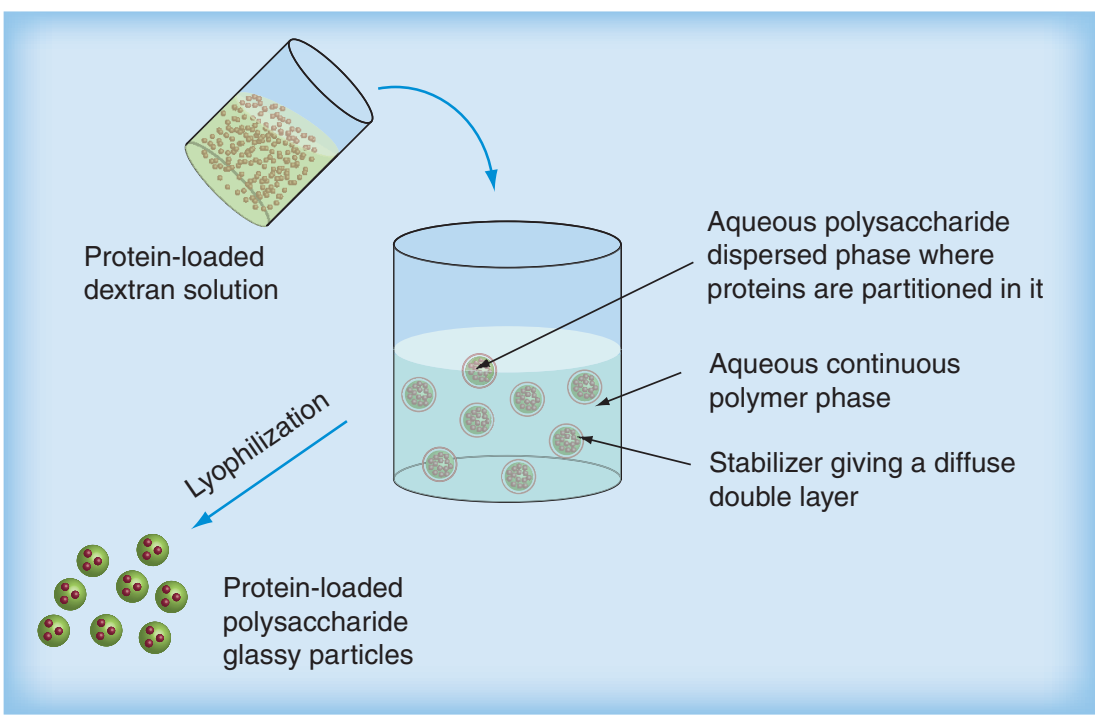

Figure 2. Loading proteins into polysaccharide particles via aqueous-aqueous emulsion.

Reproduced with permission from [2]. 


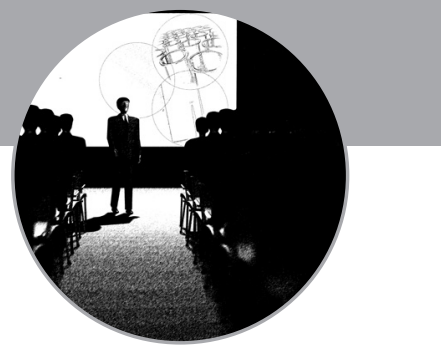

by the company (out of several products in the market) for creating diffusion channels across the epidermis prior to applying a drug-loaded patch on the pre-treated spot (Figure 3) [6]. This device possesses a patterned electric filament, which may be pressed against the skin, with pulsed electrical energy applied to ablate a layer of stratum corneum. This ablation may create some thinner spots on the stratum corneum which function as diffusion channels for proteins applied (FIGURE 3). In addition to efficacy, the speaker claimed that there was little visual evidence of poration left $24 \mathrm{~h}$ after removal of the patch.

Tuo Jin (Shanghai Jiao Tong University) introduced their newly invented patch system, phase-transition hydrogel microneedle patch, for transdermal delivery of insulin. This microneedle patch is formed of hydrophilic polymers comprising polyvinyl alcohol (PVA) blended with one or some polysaccharides. The microneedles of this patch are sufficiently hard and tough to penetrate the epidermis at dry state but turn to a hydrogel state, permeable to protein and peptide drugs (such as insulin) loaded in the polymer matrix, by absorbing body fluid in the dermis layer. The fabrication process of this PVA-based microneedle patch comprises adding biologic therapeutics in an aqueous solution of the polymers dissolved in hot water but cooled down to room temperature, casting the solution to a ceramic mold having microholes on one side, detaching from the mold, and drying. Some of the PVA chains form small crystalline domains distributed in the matrix of the polymer, which function as crosslinking junctions to hold the polymer network at a hydrated state. The polysaccharides blended in the PVA matrix may play multiple roles in the

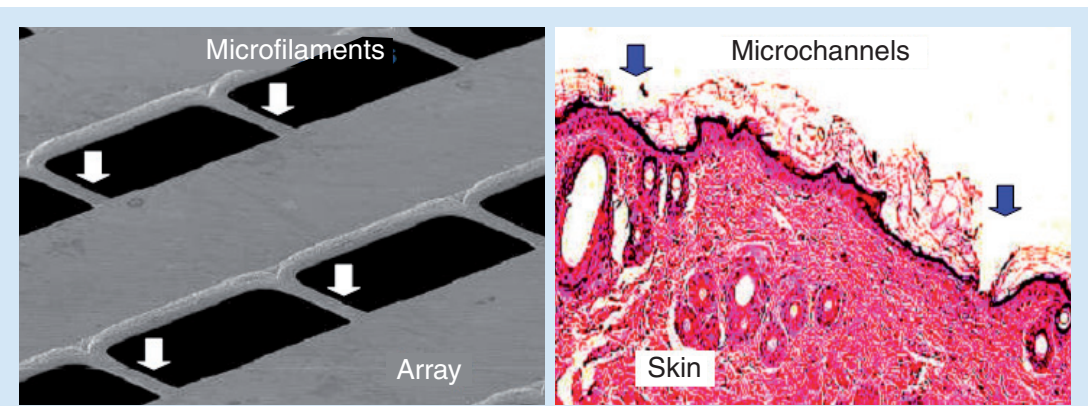

- Milliseconds pulse of electrical energy across resistive filaments

- Thermal energy conducts into, and ablates, stratum corneum

- Transient portals for delivery of water-soluble drugs/vaccines

- Painless, no sensation of heat

Figure 3. Micropores formed by thermal microablation.

Reproduced with permission from [6]. microneedle patch such as hardness enhancers, protein/peptide stabilizers, cross-linking junction diluters and diffusion channel creators. At the end of patching, the entire patch may easily be removed from skin without deposition of needle materials (such as soluble polysaccharide microneedles). A shortfall of this system may be that proteins substantially larger than insulin in molecular weight may not be released smoothly through the swollen PVA network.

Instead of delivery systems, William Frey II (Alzheimer's Research Center at Regions Hospital St Paul, MN, USA) presented his groups' study on administration of a variety of biologic therapeutics (proteins/peptides and stem cells) through the intranasal route [7]. The intranasal route is especially efficient for delivering therapeutics to the brain and CNS system by bypassing the blood-brain barrier. The speaker provides some case studies of using the intranasal route to deliver proteins, nucleotides and steam cells to treat tumors, stroke and Alzheimer's disease.

One of the two lectures on responsive hydrogels, local (inner ear) administration of thermal gelling Poloxamer 407 formulation, was described by Qiang Ye (Otonomy, Inc., CA, USA). An aqueous solution of Poloxamer 407 gelled sharply at the phase transition temperature, which was a function dependent on the concentration of the polymer solution. The transition temperature dropped from $28-21^{\circ} \mathrm{C}$ when the polymer concentration increased from $15.0-17.0 \%$. This concentration dependence may offer a great convenience in formulating a hydrogel-based sustained drug release.

Another lecture regarding responsive hydrogel systems was prepared by Kinam Park (Purdue University, IN, USA) but delivered by Qiang Ye. Park and Ye reviewed a number of responsive hydrogel materials developed by a variety of teams, named 'smart hydrogel' or 'extremely smart hydrogel' [8], not only in attempts to introduce technological and mechanistic details but also stressing translational feasibility. The lecturers suggested turning new material-directed hydrogel research towards the physiological pathway and therapeutic need-directed hydrogel research (Figure 4) [9].

\section{Analytical technologies}

The analytical section featured three presentations, two by Jeff Mazzeo from (Waters, MA, USA) and one by Karen Miller (Amgen, CA, USA). Mazzeo's first talk was a summary of key analytical methodologies used in the 
characterization of protein biopharmaceuticals. The main focus was on methods that employ liquid chromatography and mass spectrometry, including peptide mapping, intact mass measurement, aggregation analysis by size exclusion chromatography, charge-variant analysis by ion exchange chromatography and higherorder structure analysis by hydrogen-deuterium exchange mass spectrometry (HDX-MS).

The latest advances in each of these areas were covered, including the use of ultra performance liquid chromatography (UPLC) systems and chemistries for higher resolution, sensitivity and throughput, accurate mass measurements for confident identification of peptides and proteins, MS/MS for sequencing of peptides, and the capabilities of the HDX-MS method for looking at changes in conformation. Mazzeo stressed the need for good software tools to turn acquired data into knowledge in the most efficient way. All modern LC/MS systems are capable of producing high quality data, but turning that data into actionable information relies on advanced software tools that automate data analysis and report as well as facilitate comparisons between samples.

In Mazzeo's second talk he focused on workflows for characterization of glycosylation, including: the ability to rapidly screen glycoforms using intact mass measurements; more in-depth analysis of the major and minor glycans after enzymatic release of the glycans and subsequent derivatization with fluorophores and high resolution UPLC analysis coupled with MS for confident identification; and site-specific glycosylation analysis at the glycopeptide level by both reversed-phase chromatography as well as hydrophilic interaction chromatography, which allows isolation of the glycopeptides from nonglycopeptides, in both cases coupled with mass spectrometry for glycan structure information as well as site of attachment.

Miller discussed the challenges associated with the characterization of PEGylated proteins, which arise from the heterogeneity of PEG as well as the potential for multiple sites of attachment. Miller stressed the importance of full characterization of the non-PEGylated protein as the first step in the characterization process. Peptide mapping with proteolytic enzymes that lead to larger fragments was demonstrated to be quite useful for determining the site of PEGylation. Reversed phase analysis was found to be useful for looking at the level of PEGylated protein versus non-PEGylated protein, as was SDS-PAGE. The challenges of obtaining an accurate mass measurement of the PEGylated protein were highlighted, with the potential of newer methods to facilitate this analysis mentioned. The latter part of Miller's talk focused on a risk assessment model for PEGylated proteins.

\section{Regulatory landscape \& biosimilars}

The first talk by Ravi Harappanhalli, Principal Consultant with Paraxel (Washington DC, USA), provided a summary of the regulations applicable to recombinant DNA-derived protein products from expression systems such as bacteria, yeast, insect cells, mouse and $\mathrm{CHO}$ cells. To file these products in a biological license application (BLA) these products need to be tested by a battery of methods that will monitor for process-related and product-related impurities/ related substances. Common process-related impurities include residual processing reagents, host cell proteins, residual DNA and protein A. Product-related impurities are generated by the degradation pathways for proteins including deamidation, oxidation, aggregation, fragmentation and disulfide bond shuffling. Size-exclusion chromatography and analytical ultracentrifugation are two methods commonly used to test for aggregates, and ion-exchange chromatography is used to monitor for charge variants. Reversed phase liquid chromatography and hydrophobic interaction chromatography are other methods used for purity testing. Peptide mapping is currently the premier identity method and together
Current
Future

Material development: Smart hydrogels with high IQ

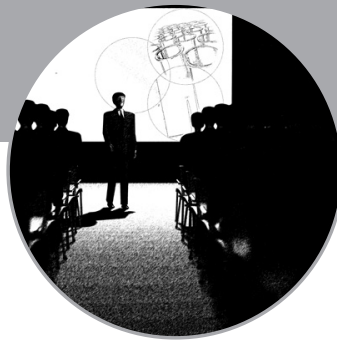


with mass spectroscopy is used for sequence confirmation. The product specifications, which include the above listed methods, are set early in the drug development process based on a limited amount of available lot release and stability data. The specification limits are optimized after licensure, as additional product data become available. Any manufacturing changes will require a comparability study that may include physiochemical testing, functional assays, nonclinical studies and clinical studies although in many cases, comparability may be deduced exclusively from quality studies. Tests included in the lot release and stability testing are usually not sufficient to establish comparability.

The second topic of the day was Biosimilars Regulatory Landscape by Nanda Subbarao, Senior Consultant with the Biologics Consulting Group (NY, USA). The BPCI Act of March 2010 established an abbreviated pathway for the registration of biosimilar products in the USA. The FDA held a public hearing on the implementation of this act in November 2010 and continues to accept feedback. In the meantime, guidelines for biosimilars have been published by multiple regional authorities. The EU has overarching guidances covering all products and additional ones addressing individual products such as insulin and G-CSF. The WHO has guidance for the individual regional authorities, which may implement biosimilar guidances within their region. The existing guidances still continue to be discussed and are likely to be revised as new challenges emerge and the industry and regulatory authorities acquire experience in this area. For example, biosimilar companies face the challenge of having to perform comparability studies against different, often very similar, reference products in order to file the biosimilar in different global areas. Regulations that decrease such unnecessary studies will lead to resource savings as well as avoid unethical repetitions of animal and/or clinical studies. A second challenge is presented by the need to define appropriate acceptance criteria for PK studies; we expect clearer guidances on this topic as the regulatory authorities acquire experience with biosimilars. Another challenge is presented by the current International Nonproprietary Name (INN) system. More than one biosimilar could have the same INN number although biosimilars from different manufacturers are not interchangeable. The WHO does require that prescriptions for biosimilars not be based on just the INN number to enable phamacovigilence and to ensure traceability. Nevertheless, there are on-going discussions in the industry as to whether the INN system should be revised for biosimilars. The reference product manufacturer will continue to make manufacturing improvements resulting in lower impurity levels. The process by which the biosimilar will remain comparable to this moving target is not understood. For all these reasons it is expected that the regulations will continue to evolve over the coming years.

The final talk of the session by Harappanhalli addressed development strategies for biosimilars. It is clear that the typical development process used for small molecule generics cannot be used for biosimilars because proteins are complex in structure and have a much higher level of heterogeneity when compared with small molecules and these factors can strongly impact the potency of the drug. The complex impurity profile, the immunogenicity and the reliance of the quality on consistent and reliable process places much greater demands on the development of biosimilars. To paraphrase the EU guideline, it is not expected that the quality attributes will be identical to that of the reference product. However, larger differences or uncertainties in the differences will necessitate more extensive preclinical and clinical studies. The larger and more complex proteins, such as mAbs, are expected to present the greatest challenge for development of biosimilars and currently blood vaccines and cell products are not considered too complex and are therefore suitable for biosimilars at this time.

\section{Conclusion}

This year's Arden Conference differed from previous ones in that it was focused on the industrial development of biological therapeutics, a type of pharmaceutical product highly 'dynamic' compared with small-molecule drugs. The challenges raised in the areas covered by this workshop (preformulation, manufacturing process, delivery, analysis as well as regulatory landscape) were all associated with a common factor, conformational instability of proteins. The strategies demonstrated for addressing the challenges comprised both 'textbook' type of approaches and 'research' type suggestions. The latter case was especially true in the session of advanced delivery systems and the topics regarding development of 'biosimilars' (which was discussed in several sessions including manufacturing, analysis and regulation). Nevertheless, the conference served as an excellent one-stop-course for industrial and academic attendees to update their knowledge regarding development of protein therapeutics. 


\section{Acknowledgements}

The authors thank the conference lecturers who kindly agreed to share their slides with readers of this report.

\section{Financial \& competing interests disclosure}

The authors have no relevant affiliations or financial involvement with any organization or entity with a financial interest in or financial conflict with the subject matter or materials discussed in the manuscript. This includes employment, consultancies, honoraria, stock ownership or options, expert testimony, grants or patents received or pending, or royalties.

No writing assistance was utilized in the production of this manuscript.

\section{Bibliography}

1 Shire S. Aggregation and potential impact on safety and physical properties of protein biotherapeutics. Presented at: AAPS 46th Arden Conference: Pharmaceutical Development of Biologics: Fundamentals, Challenges and Recent Advances. West Point, NY, USA, 6-11 March 2011.
2 Jin T, Yuan WE, Hu ZH. Die-hard challenges in sustained-release protein formulations: reducing initial burst and maintaining drug conformational stability simultaneously. Presented at: AAPS 46th Arden Conference: Pharmaceutical Development of Biologics: Fundamentals, Challenges and Recent Advances. West Point, NY, USA, 6-11 March 2011.

3 Jin T, Zhu JH, Zhu H et al. Preparing polymer-based sustained-release systems without exposing proteins to water/oil or water/air interfaces and cross-linking reagents. J. Control. Release 128, 50-59 (2008).

4 Perlin DS. Amphotericin B cochleates: a vehicle for oral delivery. Curr. Opin. Investig. Drugs 5, 198-201 (2004).

5 Lambert WJ, Los K. DepoFoam ${ }^{\circledR}$ multivesicular liposomes for the sustained release of macromolecules. In: ModifiedRelease Drug Delivery Technology (Second Edition). Rathbone MJ, Roberts MS (Eds). Informa Healthcare, 2, 207-214 (2008).

6 Tomlinson E. Recent advances in transdermal delivery of biologics and major hurdles to commercialization. Presented at: AAPS 46th Arden Conference: Pharmaceutical

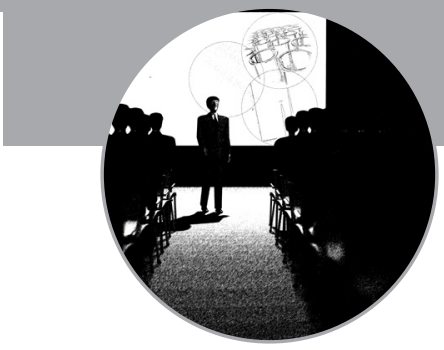

Development of Biologics: Fundamentals, Challenges and Recent Advances. West Point, NY, USA, 6-11 March 2011.

7 Thorne RG, Pronk GJ, Frey II WH et al. Delivery of insulin-like growth factor-I to the rat brain and spinal cord along olfactory and trigeminal pathways following intranasal administration. Neuroscience 127, 481-496 (2004).

8 Betancourt T, Peppas LB. Micro- and nanofabrication methods in nanotechnological medical and pharmaceutical devices. Int. J. Nanomed. 1, 483-495(2006).

9 Ye Q, Park K. Recent advances in hydrogel drug delivery for biotherapeutics and major hurdles to commercialization. Presented at: AAPS 46th Arden Conference: Pharmaceutical Development of Biologics: Fundamentals, Challenges and Recent Advances. West Point, NY, USA, 6-11 March 2011. 